\title{
High-density Hyaluronic Acid for the Treatment of HIV-related Facial Lipoatrophy
}

\author{
Marco Pignatti • Antonio Pedone • Alessio Baccarani • \\ Giovanni Guaraldi · Gabriella Orlando · \\ Matteo Lombardi · Giorgio De Santis
}

Received: 29 March 2011/ Accepted: 2 June 2011

(c) Springer Science+Business Media, LLC and International Society of Aesthetic Plastic Surgery 2011

\begin{abstract}
Background Facial lipoatrophy is a stigmatizing hallmark of HIV. The injection of facial fillers has an essential role in the treatment of this condition. The objective of our study was to verify the safety and efficacy of a new formulation of high-density hyaluronic acid for the injectable treatment of HIV-related facial lipoatrophy.

Methods We treated with high-density hyaluronic acid injections HIV patients affected by moderate to severe facial lipoatrophy and evaluated them at last follow-up, at a minimum of 36 weeks. Physician-related outcomes included pre-and post-treatment ultrasound measurement of the soft-tissue thickness of the cheeks and qualitative assessment of aesthetic results by means of the Global Aesthetic Improvement Scale using pre- and post-treatment photos of the patients. Patient satisfaction outcomes were evaluated with the VAS-face scale and Freiburg test.

Results Fifty-four patients were studied. The median number of treatment sessions was 3 and the median length of treatment was 5.5 months. The thickness of the soft tissues of the cheek increased significantly from 9.45 to $13.12 \mathrm{~mm}$ $(p<0.0001)$. On the basis of the Global Aesthetic Improvement Scale, $87.5 \%$ of the patients were judged as
\end{abstract}

\footnotetext{
M. Pignatti $(\bowtie) \cdot$ A. Pedone - A. Baccarani - M. Lombardi · G. De Santis

Department of Plastic and Reconstructive Surgery, Policlinico di Modena, University of Modena and Reggio Emilia, Modena, Italy

e-mail: mrpignatti@googlemail.com

G. Guaraldi · G. Orlando

HIV Metabolic Clinic, Department of Infectious and Tropical Diseases, University of Modena and Reggio Emilia, Modena, Italy
}

"much improved" or "improved." Patient satisfaction at 1 year from the end of treatment was proven (VAS-face: 77.9; Freiburg questionnaire: $93.6 \%$ of patients were satisfied or very satisfied). Complications were limited to mild redness and swelling in the early postoperative period.

Conclusion Long-term improvement of facial contour and excellent patient satisfaction, in the absence of severe side effects, were obtained by the injection of high-density hyaluronic acid (STYLAGE ${ }^{\circledR}$ XL) in HIV patients with facial lipoatrophy.

Keywords Hyaluronic acid · Facial fillers · High density · Facial lipoatrophy $\cdot$ STYLAGE $^{\circledR}$ XL

HIV-associated lipodystrophy is a syndrome characterized by body fat changes, including loss of peripheral fat tissue (lipoatrophy) typically in the limbs, buttocks, and the face (facial lipoatrophy, FLA), and frequently, but not necessarily, associated with central fat accumulation (lipohypertrophy). The etiology of the syndrome, although still not completely clarified, includes antiretroviral therapy (ART), HIV per se, genetic predisposition, immunologic/inflammatory response, and metabolic dysregulation [1]. Lipoatrophy is still highly prevalent among these patients even if the incidence is decreasing thanks to a modification in the use of the ART drugs [2-4]. Nevertheless, in the FRAM case control study (Fat Redistribution And Metabolic change in HIV infection) HIV-associated lipoatrophy was shown to persist for more than 5 years of follow-up [5].

Facial lipoatrophy (FLA) is a stigmatizing feature of HIV patients and may compromise their quality of life [6], causing severe psychological distress, body image impairment, loss of self-esteem, depression, social isolation, sexual dysfunctions, and career barriers [7]. The thymidine analog 
ART-sparing regimens did not prove to be sufficient, by themselves, to treat facial lipoatrophy [5]. The injection of facial fillers, has, therefore, an important role as a complementary therapeutic intervention [3]. Commonly used fillers include autologous fat on one side and permanent or absorbable synthetic materials on the other. Among the permanent fillers, polymethylmethacrylate, expanded polytetrafluoroethylene implants, polyacrylamide gel, and other synthetic injectable polymers have been used [8]. Among absorbable fillers are polylactic acid [9], calcium hydroxyapatite, collagen, Alloderm, and hyaluronic acid [1]. Only two partially randomized studies have been published that compare different fillers so it is not yet established which treatment is more adequate $[10,11]$.

Hyaluronic acid (HA) is the filling material most widely used $[12,13]$, especially in cosmetic surgery. It is US FDAapproved for the correction of facial wrinkles and folds (Restylane, Medicis Aesthetics, Scottsdale, AZ, USA; and Hylaform, Inamed Aesthetics, Santa Barbara, CA, USA). It is safe, has a low complication rate [14], and has the advantage that its effects are reversible [15]. To increase durability of volume correction while keeping a low incidence of side effects, large particle and high-density hyaluronic acid has been marketed $[14,15]$. The use of these long lasting hyaluronic acid (Restylane SubQ2, and Juvaderm Voluma, Allergan, Irvine, CA, USA) has recently been reported for the treatment of HIV facial lipoatrophy [16-18].

The objective of our study was to describe the safety and effectiveness of a new formulation of high-density hyaluronic acid filler for the treatment of moderate to severe HIV-related facial lipoatrophy in a large series of patients followed for a long period of time.

\section{Methods}

An observational study of a series of consecutive patients treated with HA facial injections for moderate to severe, as defined by Fontdevila [19], HIV-related facial lipoatrophy and who reached a minimum of 36 weeks of follow-up was conducted at the Metabolic Clinic of the University of Modena and Reggio Emilia. This is a tertiary-level referral center of the Public National Health System where patients receive, free of charge, comprehensive metabolic and anthropometric diagnostic and therapeutic assessments for the presence of lipodystrophy and HIV-associated comorbidities. The multidisciplinary team includes infectious disease specialists, cardiologists, endocrinologists, radiologists, nutritionists, personal trainers, psychologists, and plastic surgeons [20].

Inclusion criteria for the study were HIV-related moderate to severe facial lipoatrophy, adult age, exposure to highly active antiretroviral therapy for at least 6 months, platelet count greater than 50,000 cells/L, a minimum of 36 weeks of follow-up from the end of the injection treatment, and consent to be part of the study. Exclusion criteria were previous use at the same site of permanent facial fillers or injection of absorbable fillers within the previous 6 months, pregnancy, and localized infections of the face.

A high-density $(26 \mathrm{mg} / \mathrm{g}) \mathrm{HA}$ of nonanimal origin (STYLAGE ${ }^{\circledR}$ XL, Vivacy Laboratoires, Paris, France) was used. This filler is declared by the manufacturer to be absorbed in more than 12 months and is indicated to increase soft tissue volume in any area of the body. The product is made of two separately reticulated molecular chains (tight ones and loose ones), interpenetrated with crosslinks that are not chemical bonds but relatively weak interactions between the two structures (IPN-like technology $=$ InterPenetrated Network), to obtain a dense texture. The overall structure is monophasic. This composition is supposed to reduce degradation by hyaluronidase and heat, therefore increasing durability.

Each patient was treated with the injection of one vial (1 ml) of STYLAGE XL in each cheek at each monthly outpatient appointment, until a correction of the facial soft tissue defect was considered satisfactory by the surgeon, the infectious disease specialist, and the patient. The injection was performed with a $271 / 2$-gauge or a 25 -gauge needle in the subcutaneous tissue using the linear threading technique and either a fanning or a cross-radial distribution or both, depending on the patient's needs [21]. At the end of each treatment, the injected areas were gently massaged to optimize the result.

\section{Physician-related Outcomes}

Standardized photographs of the face were taken and ultrasound of the cheek soft tissues was performed in all patients before starting therapy and at weeks 12, 24, 36, and 60. Ultrasound examination of the cheeks using a 7.5$\mathrm{MHz}$ probe was used to record dermal and subcutaneous thickness, assessed as the average of three readings taken perpendicular to the skin surface at the nasolabial fold, at the angle of the mouth, at the zygomatic arch, and centrally between these points in the area of the buccal fat pad. Final ultrasound thickness was evaluated at the end of follow-up and compared with the pre-treatment measurements. To evaluate the effect of a single treatment session, the results of the same measurements after the same follow-up interval were evaluated separately in a subgroup of patients who had received only one treatment.

The pre- and post-treatment photos at 1 year were evaluated by three head and neck surgeons (who had not performed the treatment), three nurses, and three nonmedical 
persons. They were requested to judge the appearance of the patients and express their opinion according to the Global Aesthetic Improvement Scale (GAIS): "very much improved," "much improved," "improved," "no change," or "worse" [22].

\section{Patient-related Outcomes}

Patient satisfaction was evaluated by means of the visual analog scale (VAS)-face and the Freiburg questionnaire. According to VAS for the face, patients were asked how they judged their appearance at the time of interview, expressed as a number from 0 to 100 , where 100 was the best possible appearance. On the basis of the Freiburg questionnaire on aesthetic dermatology and cosmetic surgery, patients were asked whether the treatment adequately corrected the defects of their faces and whether they would suggest this treatment to other patients with the same problem [18].

\section{Statistical Analysis}

The differences of the cheek dermal and subcutaneous thicknesses measured by ultrasound at baseline and at final follow-up were analyzed by the Mann-Whitney $U$ test for the whole cohort and separately for the subgroup which underwent only one treatment session. Qualitative results of outcomes as judged by physicians and patients were analyzed using the $\chi^{2}$ test. All statistical analyses were performed using STATA 10.1 Intercooled (statistics/data analysis) software for Mac (Stata Corp., College Station, TX, USA).

\section{Results}

Fifty-four (41 males) of the 379 patients treated with at least one hyaluronic acid injection from June 2008 to December 2009 reached at least 36 weeks of follow-up and were analyzed in this study. At baseline, the median age was 47 years (range $=38.4-62.76$ ) and median BMI was 22.27. The median number of treatment sessions was 3 (range $=1-9$ ) and median length of treatment was 5.5 months. Ultrasound measurements showed a median increase in cheek thickness (dermis plus subcutaneous tissue) of $3.67 \mathrm{~mm}$ from a pretreatment median value of $9.45 \mathrm{~mm}$ (range $=0-15.1$ ) to a post-treatment median value of $13.12 \mathrm{~mm}$ (range $=$ 8.75-18.85) $(p<0.001)$ at a minimum follow-up time of 36 weeks (Fig. 1).

In the subgroup of 12 patients who received only one HA injection ( $1 \mathrm{ml}$ in each cheek) and who were analyzed separately, there were 8 males and 4 females with a median age of 47.2 years $\quad($ range $=39.9-62.33)$. The cheek

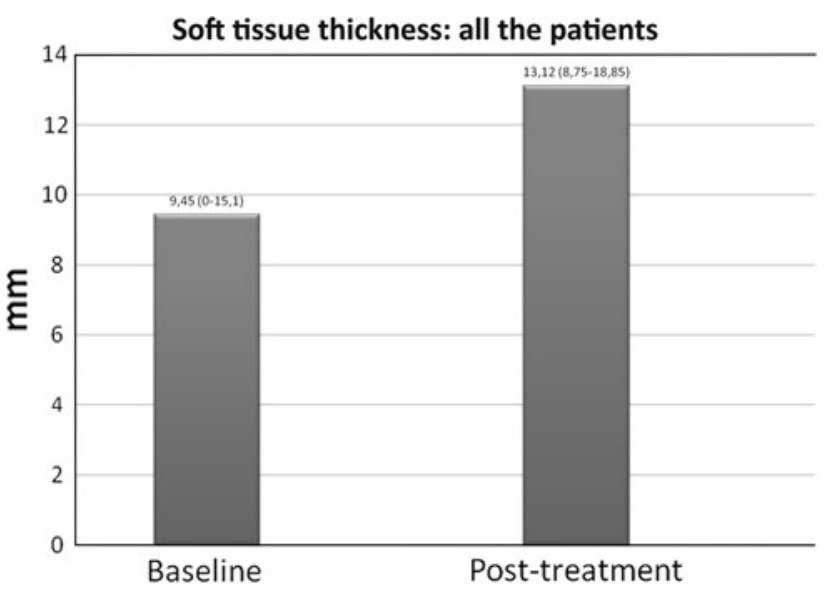

Fig. 1 Soft-tissue thickness of the cheeks of all the patients in the study evaluated with ultrasound. Statistically significant improvement of thickness at follow-up

thickness improved from a pretreatment median value of $7.82 \mathrm{~mm}$ (range $=0-14.15)$ to a post-treatment median value of $12.25 \mathrm{~mm}$ (range $=9.4-15.75),(p=0.0163)$ (Fig. 2). The median BMI was 22.05 pretreatment and 22.56 post-treatment.

Physician-related outcome as judged by the Global Aesthetic Improvement Scale based on the pretreatment and 1-year post-treatment photos was "very much improved" in $27(50 \%)$ patients, "much improved" in 20 (37.5\%) patients, and "improved" in 7 (2.5\%) patients. No patient was considered to have worsened after the treatment.

Patient-related outcome, analyzed by the VAS-face test in a sample of 47 patients, was very good (77.9). Answering the Freiburg questionnaire, 9 patients (19.1\%) declared the results to be very satisfactory, 35 patients

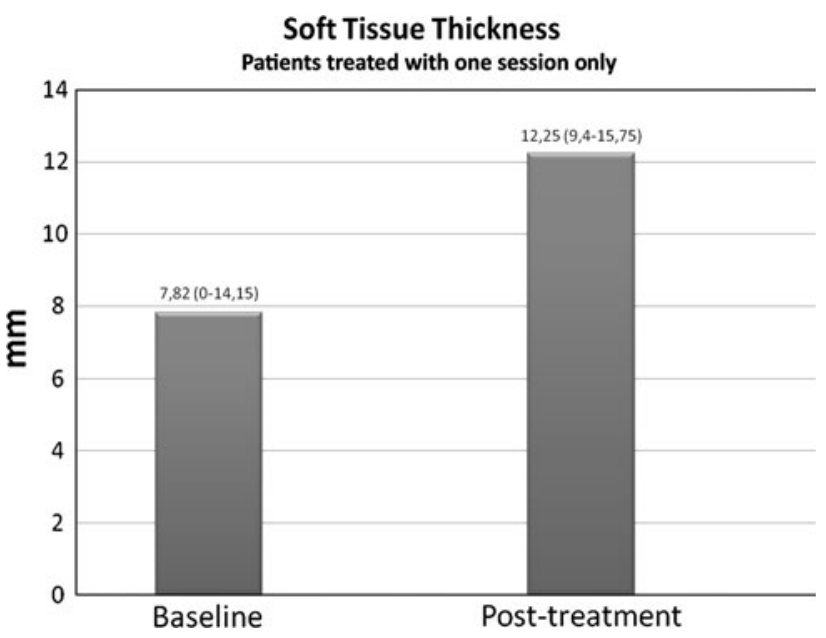

Fig. 2 Soft-tissue thickness of the cheeks of the subgroup of patients treated with one session only. There is statistically significant longlasting improvement of soft-tissue thickness of the cheek 


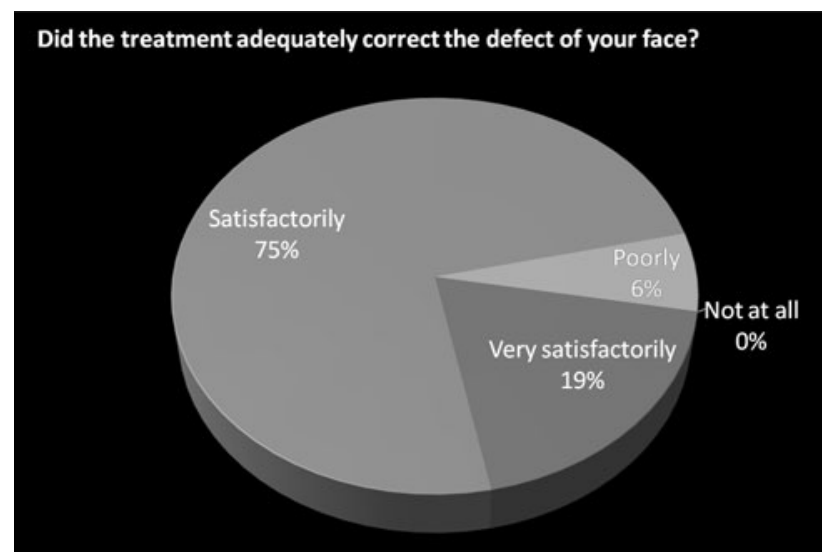

Fig. 3 Results of the patient satisfaction evaluation at 1 year from the end of treatment. Answer to the first question of the Freiburg questionnaire on aesthetic dermatology and cosmetic surgery: "Did the treatment adequately correct the defects of your face?"

(74.5\%) satisfactory, and 3 patients $(6.4 \%)$ poor. None of the patients found the results not at all satisfactory (Fig. 3).

All 47 patients $(100 \%)$ would suggest this treatment to other patients with the same problem.

Adverse events reported in the follow-up period included mild redness and swelling of different levels of severity experienced by $70 \%$ of patients in the early postoperative period. Also, tenderness and pain of low intensity was reported only in the early postoperative period by 11 patients (20.4\%). No patient developed a fever. Ten $(18.5 \%)$ patients with severe FLA complained, at the time of the second treatment session, of the presence of nonvisible but palpable lumps. These could be attributed to subcutaneous deposits of hyaluronic acid in the very thin soft tissue layer which is typical at the beginning of the treatment. The subsequent injections in the surrounding hypotrophic areas corrected this perceived abnormality.

No patient developed skin indurations. One patient developed a sudden swelling of the treated area after a flulike episode 3 months after the end of treatment. However, no redness or fluid collections were reported and no medical advice was sought. The reported swelling resolved spontaneously in 2 weeks. No infections or other complications were encountered.

Figures 4, 5, and 6 show the pre- and post-treatment photos of patients suffering from FLA of different severity levels.

\section{Discussion}

Facial lipoatrophy in HIV patients has been treated previously with the injection of permanent or absorbable materials, including hyaluronic acid, with satisfactory results [1, $8,9,17,18,23]$. The obvious drawback of absorbable fillers is the need to repeat treatments over time. Recent studies have shown that large-particle and high-density hyaluronic acid preparations (Restylane SubQ, Juvaderm Voluma) are able to safely and efficiently provide correction of the tissue defect in this kind of patient for a longer time (up to 12 months post-treatment) than the hyaluronic acid preparation of usual density [16-18].

The efficacy of high-density hyaluronic acid was confirmed in our study by the statistically significant improvement in thickness of the dermis-subcutaneous layer of the cheeks. The BMI variation between the beginning and the end of treatment, on the other hand, was not statistically significant and could not be considered responsible for the change. Our results confirmed also the long-lasting efficacy
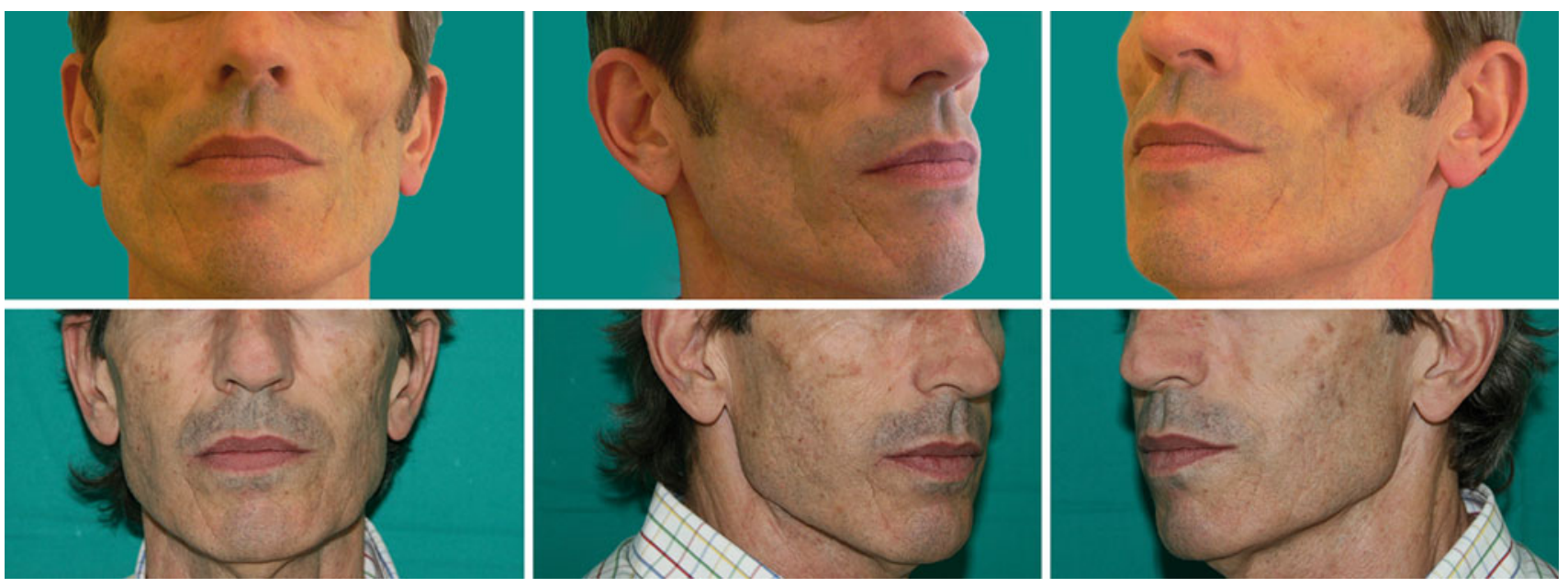

Fig. 4 Photos of a patient suffering from severe FLA. He received six treatment sessions, had a VAS satisfaction of 90, and no complications. Judged at GAIS (Global Aesthetic Improvement Scale) as "much improved." Top row Pretreatment. Bottom row At 13 months of follow-up 

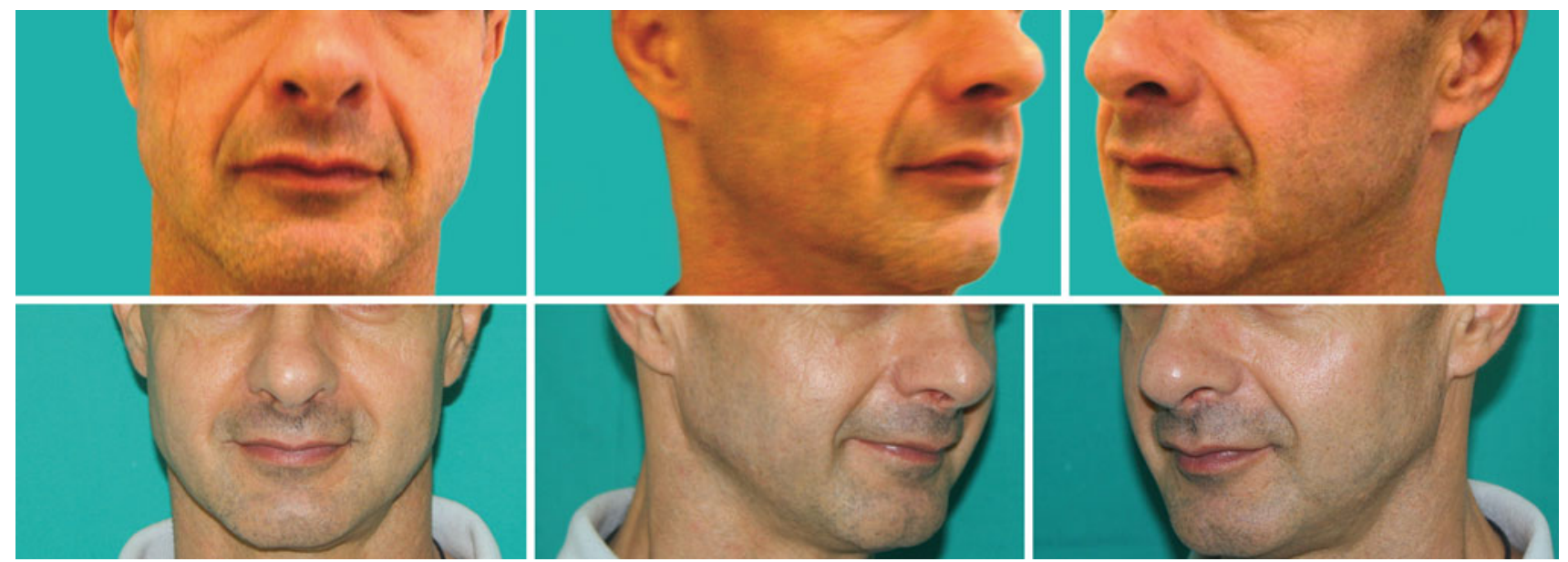

Fig. 5 Photos of a patient suffering from moderate FLA. He had three treatment sessions and had a VAS satisfaction of 80. Judged at GAIS (Global Aesthetic Improvement Scale) as "much improved." Top row Pretreatment. Bottom row At 14 months of follow-up
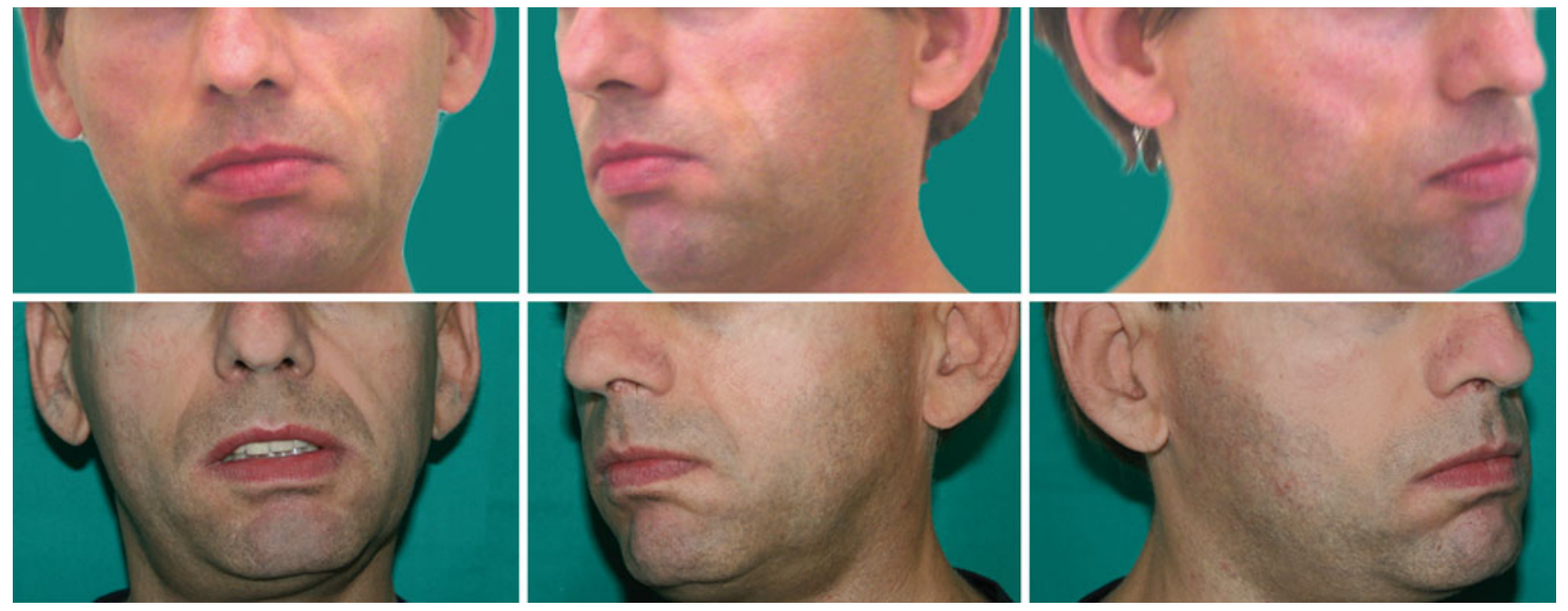

Fig. 6 Photos of a patient suffering from moderate FLA who had one treatment session, had a VAS satisfaction of 100 , and no complications. Although a noticeable improvement is seen by the authors, this patient was judged at the GAIS (Global Aesthetic Improvement

of high-density hyaluronic acid, the rarity of complications, and the satisfaction of the patients $(93.6 \%$ of 47 patients at 1 year after the end of treatment).

Interestingly, a small number of treatment sessions appeared to be sufficient to correct the defect. Depending on the level of severity of FLA and on the self-perception of each patient, however, the number of treatment sessions can vary. In our patients between 1 and 9 sessions were necessary for optimal results.

We decided, per protocol, to inject a maximum amount of $1 \mathrm{ml}$ in each cheek at each treatment session. Although previous studies [16] have shown that the volume injected can be increased, complications such as skin indurations could also increase, reaching $23 \%$ in the study by Skeie
Scale) test to be between "improved" and "no change" from pretreatment. Top row Pretreatment. Bottom row At 12 months of follow-up

et al. [17], where up to $3 \mathrm{ml}$ of Restylane SubQ was injected into each cheek at each session. High-density HA was injected, as suggested by the manufacturer, into the subcutaneous tissue using a technique similar to the one used for the HA of lower density.

The results of high-density HA injections in the subgroup of patients who had only one treatment session suggest that even a single injection is able to increase the soft tissue thickness. A statistical analysis trying to correlate the surprisingly good results of this subgroup of patients to their demographic characteristics or therapeutic regimens was unable to show any significant correlation. The obvious limitation of these results is the small number of patients treated only once. 
No patient in our study experienced serious adverse effects. In particular, no skin indurations requiring the use of hyaluronidase, as previously described by Skeie et al. [17], were observed. The only side effects encountered were mild redness and swelling which resolved spontaneously and which are normally encountered in cosmetic practice.

In conclusion, this study shows that the injection of the high-density hyaluronic acid STYLAGE XL is able to safely and efficiently correct the soft tissue defect of facial lipoatrophy in HIV patients, with mild side effects and no major complications. Facial contour was improved for a long period of time, exceeding in several cases 12 months, with an excellent rate of patient satisfaction.

Disclosure The authors declare that they have no conflicts of interest to disclose.

\section{References}

1. Jones D (2005) HIV facial lipoatrophy: causes and treatment options. Dermatol Surg 31:1519-1529

2. Nguyen A, Calmy A, Schiffer V, Bernasconi E, Battegay M, Opravil M, Evison JM, Tarr PE, Schmid P, Perneger T, Hirschel B, Swiss HIV Cohort Study (2008) Lipodystrophy and weight changes: data from the Swiss HIV Cohort Study, 2000-2006. HIV Med 9(3):142-150

3. Benn P, Sauret-Jackson V, Cartledge J, Ruff C, Sabin CA, Moyle G, Linney A, Reilly G, Edwards SG (2009) Improvements in cheek volume in lipoatrophic individuals switching away from thymidine nucleoside reverse transcriptase inhibitors. HIV Med 10(6):351-355

4. Hammond E, McKinnon E, Nolan D (2010) Human immunodeficiency virus treatment-induced adipose tissue pathology and lipoatrophy: prevalence and metabolic consequences. Clin Infect Dis 51(5):591-599

5. Grunfeld C, Saag M, Cofrancesco J Jr, Lewis CE, Kronmal R, Heymsfield S, Tien PC, Bacchetti P, Shlipak M, Scherzer R (2010) Study of fat redistribution and metabolic change in HIV infection (FRAM). Regional adipose tissue measured by MRI over 5 years in HIV-infected and control participants indicates persistence of HIV-associated lipoatrophy. AIDS 24:1717-1726

6. Echavez M, Horstman W (2005) Relationship between lipoatrophy and quality of life. AIDS Read 15:369-375

7. Guaraldi G, Luzi K, Murri R, Granata A, De Paola M, Orlando G, Squillace N, Malmusi D, Carani C, Comelli D, Esposito R, Martinez E (2007) Sexual dysfunction in HIV-infected men: role of antiretroviral therapy, hypogonadism and lipodystrophy. Antivir Ther 12(7):1059-1065

8. De Santis G, Jacob V, Baccarani A, Pedone A, Pinelli M, Spaggiari A, Guaraldi G (2008) Polyacrylamide hydrogel injection in the management of human immunodeficiency virusrelated facial lipoatrophy: a 2-year clinical experience. Plast Reconstr Surg 121:644-653
9. Valantin MA, Aubron-Olivier C, Ghosn J, Laglenne E, Pauchard M, Schoen H et al (2003) Polylactic acid implants (New-Fill) to correct facial lipoatrophy in HIV-infected patients: results of the open-label study VEGA. AIDS 17:2471-2477

10. Guaraldi G, Orlando G, De Fazio D, De Lorenzi I, Rottino A, De Santis G, Pedone A, Spaggiari A, Baccarani A, Borghi V, Esposito R (2005) Comparison of three different interventions for the correction of HIV-associated facial lipoatrophy: a prospective study. Antivir Ther 10(6):753-759

11. Negredo E, Higueras C, Adell X, Martinez JC, Martinez E, Puig J, Fumaz CR, Muñoz-Moreno JA, Perez-Alvarez N, Videla S, Estany C, Cinquegrana D, Gonzalez-Mestre V, Clotet B (2006) Reconstructive treatment for antiretroviral-associated facial lipoatrophy: a prospective study comparing autologous fat and synthetic substances. AIDS Patient Care STDS 20(12):829-837

12. Carruthers JDA, Glogau RG, Blitzer A et al (2008) Botulinum toxin type A, hyaluronic acid dermal fillers, and combination therapies-consensus recommendations. Plast Reconstr Surg 121(Suppl 5):5S-30S

13. Redbord KP, Busso M, Hanke CW (2011) Soft-tissue augmentation with hyaluronic acid and calcium hydroxyl apatite fillers. Dermatol Ther 24(1):71-81

14. Friedman PM, Mafong EA, Kauvar AN, Geronemus RG (2002) Safety data of injectable nonanimal stabilized hyaluronic acid gel for soft tissue augmentation. Dermatol Surg 28:291-294

15. Beasley KL, Weiss MA, Weiss RA (2009) Hyaluronic acid fillers: a comprehensive review. Facial Plast Surg 25(2):86-94

16. Bugge H, Negaard A, Skeie L, Bergersen B (2007) Hyaluronic acid treatment of facial fat atrophy in HIV-positive patients. HIV Med 8:475-482

17. Skeie L, Bugge H, Negaard A, Bergersen BM (2010) Large particle hyaluronic acid for the treatment of facial lipoatrophy in HIV-positive patients: 3-year follow-up study. HIV Med 11: 170-177

18. Bechara FG, Gambichler T, Brockmeyer NH, Sand M, Altmeyer P, Hoffmann K (2008) Hyaluronic acid new formulation: experience in HIV-associated facial lipoatrophy. Dermatology 217(3): 244-249

19. Fontdevila J, Prades E, Pujol T, Guisantes E, Serra-Renom JM, Gatell J, Martínez E (2007) Validation of a simple classification for facial lipoatrophy in HIVinfected adults. Antivir Ther 12(Suppl 2):L31

20. Guaraldi G, Orlando G, Squillace N et al (2006) Multidisciplinary approach to the treatment of metabolic and morphologic alterations of HIV-related lipodystrophy. HIV Clin Trials 7:97-106

21. Rohrich RJ, Ghavami A, Crosby MA (2007) The role of hyaluronic acid fillers (Restylane) in facial cosmetic surgery: review and technical considerations. Plast Reconstr Surg 120(Suppl):41S

22. Silvers SL, Eviatar JA, Echavez MI, Pappas AL (2006) Prospective, open-label, 18-month trial of calcium hydroxylapatite (Radiesse) for facial soft-tissue augmentation in patients with human immunodeficiency virus-associated lipoatrophy: one-year durability. Plast Reconstr Surg 118(3 Suppl):34S-45S

23. Moyle GJ, Lysakova L, Brown S et al (2004) A randomized open label study of immediate vs. delayed polylactic acid injections for the cosmetic management of facial lipoatrophy in persons with HIV infection. HIV Med 5:82-97 